\title{
Machine Learning Prediction of Foodborne Disease Pathogens: Algorithm Development and Validation Study
}

Hanxue Wang ${ }^{1,2}$, ME; Wenjuan Cui ${ }^{1}$, PhD; Yunchang Guo ${ }^{3}, \mathrm{PhD}$; Yi Du ${ }^{1,2}, \mathrm{PhD}$; Yuanchun Zhou ${ }^{1,2}, \mathrm{PhD}$

${ }^{1}$ Computer Network Information Center, Chinese Academy of Sciences, Beijing, China

${ }^{2}$ Chinese Academy of Sciences University, Beijing, China

${ }^{3}$ China National Center for Food Safety Risk Assessment, Beijing, China

\section{Corresponding Author:}

Yi Du, $\mathrm{PhD}$

Computer Network Information Center

Chinese Academy of Sciences

No 4, South Fourth Street

Zhongguancun, Haidian District

Beijing, 100190

China

Phone: 8615810134970

Email: duyi@cnic.cn

\begin{abstract}
Background: Foodborne diseases have a high global incidence; thus, they place a heavy burden on public health and the social economy. Foodborne pathogens, as the main factor of foodborne diseases, play an important role in the treatment and prevention of foodborne diseases; however, foodborne diseases caused by different pathogens lack specificity in their clinical features, and there is a low proportion of actual clinical pathogen detection in real life.

Objective: We aimed to analyze foodborne disease case data, select appropriate features based on analysis results, and use machine learning methods to classify foodborne disease pathogens to predict foodborne disease pathogens for cases where the pathogen is not known or tested.

Methods: We extracted features such as space, time, and exposed food from foodborne disease case data and analyzed the relationships between these features and the foodborne disease pathogens using a variety of machine learning methods to classify foodborne disease pathogens. We compared the results of four models to obtain the pathogen prediction model with the highest accuracy.

Results: The gradient boost decision tree model obtained the highest accuracy, with accuracy approaching $69 \%$ in identifying 4 pathogens: Salmonella, Norovirus, Escherichia coli, and Vibrio parahaemolyticus. By evaluating the importance of features such as time of illness, geographical longitude and latitude, and diarrhea frequency, we found that these features play important roles in classifying foodborne disease pathogens.

Conclusions: Data analysis can reflect the distribution of some features of foodborne diseases and the relationships among the features. The classification of pathogens based on the analysis results and machine learning methods can provide beneficial support for clinical auxiliary diagnosis and treatment of foodborne diseases.
\end{abstract}

(JMIR Med Inform 2021;9(1):e24924) doi: 10.2196/24924

\section{KEYWORDS}

foodborne disease; pathogens prediction; machine learning

\section{Introduction}

\section{Background}

Foodborne diseases refer to diseases caused by pathogenic factors such as harmful substances that enter the body through food intake [1]. They are usually associated with contaminated foods and pathogens or viruses contained in foods. A foodborne disease outbreak is defined as an incident in which 2 or more people experience similar diseases after consuming the same food [2]. According to a World Health Organization (WHO) report [3], 600 million people worldwide suffered from diseases 
caused by eating contaminated food every year, of whom 4.2 million die. According to the Centers for Disease Control (CDC), 48 million people are infected with foodborne diseases every year in the United States, 128,000 of whom are hospitalized and 3000 of whom die [3]. In recent years, China has also begun monitoring foodborne diseases. In 2008, 294,000 people suffered from foodborne diseases, 50,000 of whom were hospitalized and 6 died [4]. Currently, the incidence of foodborne diseases is among the highest in all kinds of diseases [5]. Frequent occurrences of foodborne diseases at home and abroad seriously endanger public health and social economy and have become an important public health and food safety issue in the world. Foodborne disease-related research and prevention efforts are urgent.

Therefore, many researchers at home and abroad study foodborne diseases, including monitoring, identification and outbreak prediction. The Foodborne Diseases Active Surveillance Network was established in the United States to monitor, track, analyze, and prevent foodborne diseases [6]. In recent years, China has also established surveillance platforms for foodborne diseases, such as the National Foodborne Disease Surveillance Reporting System [7], which classifies, stores, monitors, and statistically analyzes foodborne disease surveillance data collected nationwide. Methods for identification and diagnosis of foodborne diseases are mainly categorized into 2 types-one analyzes the molecular subtypes of pathogens using biochemical tests to diagnose foodborne diseases, another often uses statistical analysis or machine learning algorithms to identify disease information that may be included in the data [8]. For foodborne disease outbreak prediction, regression, clustering, hidden Markov model, and some timeseries prediction methods are usually used.

The main cause of foodborne diseases is that patients are infected with contaminated foods, which causes the pathogens to enter the body [9]. Therefore, research on pathogens of foodborne diseases are of great significance. However, the clinical features of foodborne diseases caused by different pathogens are not specific, and it is difficult to intuitively identify pathogens according to patient information and disease description. Traditional pathogen identification methods based on laboratory testing usually take a long time [10]. In recent years, researchers have proposed some methods for rapid detection of pathogens in foodborne diseases [11-13], including nucleic acid, immune, and biosensor methods; however, these methods require very professional equipment, and there are still some limitations in practical applications. Therefore, only a small proportion of foodborne diseases have been carried out the identification of pathogens, which greatly hinders the diagnosis of foodborne diseases and may affect doctors' ability to treat diseases caused by different pathogens and may even result in misdiagnosis. At the same time, the low proportion of foodborne pathogens identification also leads to incomplete disease data for analysis, which has a negative effect on disease burden estimation and outbreak prediction [14].

\section{Related Work \\ Foodborne Disease Analysis Based on Surveillance Platform Data}

The international community has always attached great importance to the research on foodborne diseases and has carried out many related works. The data sources for these studies include surveillance platforms, social networks, hotlines, search engines, and food samplings [15-18]; however, compared with other data sources, the data from surveillance platforms are reliable and authoritative, and the analysis results based on these data are more credible. That is because these data are usually from hospitals or health departments, and the data are all confirmed foodborne disease cases. Therefore, many foodborne disease-related surveillance platforms have been established internationally to support foodborne disease research. In 1995, the United States established the Foodborne Diseases Active Surveillance Network to monitor and track foodborne diseases [6]. The Foodborne Disease Outbreak Surveillance System is a CDC-sponsored platform for collecting information on foodborne disease outbreaks. It collects information on foodborne disease outbreaks into reports and uploads them to National Outbreak Reporting System every year [19,20]. In 2000, WHO established the Global Foodborne Infection Network for the monitoring, control and prevention of foodborne diseases. In addition, there are some other foodborne disease surveillance platforms, such as PulseNet [21] and GenomeTrakr [22]. In recent years, China has also paid attention to the surveillance of foodborne diseases. China Food Safety Risk Assessment Center established a National Foodborne Disease Surveillance Reporting System [7] to collect, store, analyze and track foodborne disease data nationwide. The data in the system contain disease case information, test information, exposed food information, and report information, which can be used for analysis and research on foodborne diseases.

These foodborne disease surveillance platforms provide a unified and authoritative source for foodborne disease data. Research on foodborne diseases using data from surveillance platforms have been popular for a long time [4,23-28]. However, most of foodborne disease research based on surveillance platform data are concentrated on statistical analysis; only a few use the data for disease aggregation analysis and outbreak prediction [29], and it has not yet been proposed to identify pathogens using surveillance platform data. As the traditional methods of pathogens' identification using biochemical testing are time-consuming and require technical support, a large proportion of the confirmed foodborne disease cases in the surveillance system have not been tested for pathogens, which will affect the subsequent estimation of foodborne disease burden and foodborne disease outbreak prediction [14]. Therefore, an accurate identification approach for foodborne pathogens based on surveillance platform data is still necessary.

\section{Foodborne Disease Analysis Based on Machine Learning}

Machine learning addresses the question of how to build computers that improve automatically through experience; it is one of the most rapidly growing technical fields [30]. In recent years, machine learning has been widely used in various fields, 
including epidemiology. Researchers propose many methods based on machine learning to diagnose diseases, predict outbreak of diseases, analyze gene of disease pathogens, and so on $[31,32]$. The successful application of machine learning in epidemiology has brought enlightenment to the study of foodborne diseases; many works have been carried out to solve foodborne disease problems using machine learning methods. In the identification of foodborne diseases, many studies choose supervised classification models as well as unsupervised clustering methods instead of traditional statistical methods [8], and it is proved that these studies can obtain good results. In the foodborne disease outbreak prediction, researchers also use machine learning methods, such as hidden Markov models [33] and DBScan models [29]. In addition, there are some works using machine learning methods to analyze foodborne pathogens. Several classification models have identified pathogens by using near infrared laser scatter images [13]. Machine learning is applied in the gene sequence analysis of foodborne pathogens, resulting in more accurate and quicker analysis [34]. The decision tree method is also used to mine the association between food, location, and pathogens based on CDC data [35].

Compared with traditional statistical analysis methods, machine learning methods can achieve more accurate result faster and can handle larger and more complex data. Therefore, machine learning methods have become popular methods to solve problems of foodborne diseases. However, most of these studies focus on the identification or prediction of diseases [8,29,31-33], and only a small part of them were carried out for the analysis of disease pathogens [13,34,35]. Often, molecular typing or gene sequence of pathogens rather than disease case information are used. There are a few machine learning-related works proposed to analyze the relationship between pathogens and disease case data from surveillance platform.

\section{Methods}

\section{Data Description}

Our data source was the National Foodborne Disease Surveillance Reporting System [7], which collected 2.6 million foodborne disease cases from 2011 to 2018. About 60,000 of them have been tested and certain pathogens have been identified, accounting for only $3 \%$ of all cases. Among the 60,000 tested cases, a total of 26 pathogens were identified, as shown in Table 1. Among them, the China Food Safety Risk Assessment Center focuses on the detection of Salmonella, Norovirus, Escherichia coli, Vibrio parahaemolyticus, and Shigella, and the first 4 pathogens (Salmonella: 26.5\%; Norovirus: $25.9 \%$; E coli: $20.9 \%$; V parahaemolyticus: $18.6 \%$ ) total more than 50,000 , accounting for $92 \%$ of the total cases, as shown in Table 1. Therefore, in the following work, we mainly focus on these 4 pathogens.

One case data entry contains information on the patient's age, gender, home address, time of illness, time of treatment, symptoms, diagnosis, and related food information (including food name, food type name, food processing type, food purchase location, and food intake location). There are also samples and sample test items related to the case, including type, number, number of strains, test method, test item category, test item name, and test result. We used pathogen types as labels. In the process of feature selection, we excluded some food and laboratory testing information. As a result, the selected features included patient's age, patient's gender, home address, time of illness, symptoms, diagnosis, food name, and food type.

We conducted exploratory data analysis to understand the feature distribution and guide data preprocessing in the subsequent step. We use the map to show the geographical distribution of the detection rate of the 4 pathogens. Some research indicated that foodborne diseases have a seasonal pattern and that climatic temperature could be a factor of incidence [36]. Therefore, we performed a visual analysis of the detection rate of the 4 pathogens by time. We also calculate the distribution of patients' age with different pathogens and visualize the distribution of patients' age. Besides, we also performed a visual analysis of the gender of the patient and the type of exposed food. The food names, symptoms, and diagnosis were textual information; therefore, they were not explored. 
Table 1. Distribution of pathogens involved in the cases.

\begin{tabular}{|c|c|}
\hline Pathogen & Count, $\mathrm{n}$ \\
\hline Salmonella & 16378 \\
\hline Norovirus & 16052 \\
\hline Escherichia coli & 12947 \\
\hline Vibrio parahaemolyticus & 11503 \\
\hline Shigella & 2004 \\
\hline Rotavirus & 1174 \\
\hline Campylobacteria & 452 \\
\hline Other pathogens & 618 \\
\hline Staphylococcus aureus & 348 \\
\hline Adenovirus & 114 \\
\hline Aeromonas hydrophila & 114 \\
\hline Star shaped virus & 112 \\
\hline Listeria monocytogenes & 97 \\
\hline Zagreb as viruses & 75 \\
\hline Vibrio cholerae & 37 \\
\hline Vibrio vulnificus & 22 \\
\hline Yersinia enterocolitica & 17 \\
\hline Bacillus cereus & 14 \\
\hline Organophosphorus & 10 \\
\hline Enterobacter sakazakii & 7 \\
\hline E coli $\mathrm{O} 157: \mathrm{H} 7 / \mathrm{NM}$ & 7 \\
\hline Other viruses & 5 \\
\hline Coliform count & 3 \\
\hline Mold count & 2 \\
\hline Hemolytic streptococcus & 2 \\
\hline Clostridium botulinum & 1 \\
\hline Rodenticide class & 1 \\
\hline Determination of total number of colonies & 1 \\
\hline
\end{tabular}

\section{Data Preprocessing}

The original data formats are described in Table 2. We mapped the 4 pathogens (Salmonella, Norovirus, $E$ coli, and $V$ parahaemolyticus) into 4 classification labels. We converted the gender data in nominal format into a binary variable, and extracted the month value from the time of illness as a time

attribute. For the age attribute, we used 10-year intervals. Home address is a distinguishable attribute, but it was stored in 3 fields (province, city and district) in the database, and each field was in numeric format. We remapped the 3 fields into text formats according to dictionaries, combined them, and calculated corresponding latitude and longitude as location attributes. 
Table 2. The original format and description of attribute data.

\begin{tabular}{lll}
\hline Attribute & Format & Description \\
\hline Pathogen name & Nominal & The name of pathogens \\
Age & Numeric & The age of patients \\
Gender & Nominal & The gender of patients \\
Sick time & Date & The time of illness \\
Province & Numeric & The value of province in patients' home address after dic- \\
City & Numeric & tionary mapping \\
District & Numeric & The value of city in patients' home address after dictionary \\
& Text & The value of district in patients' home address after dictio- \\
Symptom & Text & nary mapping \\
Diagnosis & Text & The symptom information of patients \\
Food name & Nominal & The diagnosis information of patients \\
Food type name & The name of food which patients ate \\
\hline
\end{tabular}

Symptom and diagnosis fields were in text format. Each symptom field (or diagnosis field) contained a series of symptoms (or diagnoses), separated by a comma. When we processed the symptom field, word segmentation into a set of symptoms was performed. For the diarrhea symptom, we mapped all diarrhea features that appear in the data to a dictionary. The diarrhea trait of each disease case was expressed as its corresponding value in the dictionary, the diarrhea frequency of each disease case was the value extracted from the disease case, and the diarrhea frequency of cases without diarrhea was expressed as 0 . For the vomiting symptom, we selected vomiting frequency as the attribute, and the value was in numeric format. For cases without vomiting, the frequency of vomiting was 0 . For the fever symptom, we extracted the body temperature of each disease case and divided the body temperature into 4 grades (no fever, low, medium, high). For other symptoms, we converted them into a collection of binary variables, and we set a threshold to filter out the symptoms that occur too few times. Examples of symptoms after cleaning and transforming are shown in Table 3. For the diagnosis field, we conducted word segmentation and mapped the segmented diagnose into a collection of binary variables.

Table 3. Representation of symptoms of example cases.

\begin{tabular}{lll}
\hline Symptom field & Vector & Example case 2 \\
\hline Diarrhea traits & Example case 1 & 0 \\
Diarrhea frequency & 1 & 0 \\
Fever & 5 & 1 \\
Sick & 0 & 0 \\
Hypourocrinia & 1 & 0 \\
Vomiting frequency & 1 & 3 \\
Thirst & 0 & 0 \\
Weak & 0 & 0 \\
Stomachache & 0 & 0 \\
Pale complexion & 0 & 0 \\
Tenesmus & 0 & 0 \\
Dehydration & 0 & 0 \\
\hline
\end{tabular}

The exposed food information related to the disease case included the type and name of the food. There were 23 food categories which were expressed in nominal format. We converted these into one-hot representations. We first performed data cleaning and word segmentation on the food name field. We removed punctuation, special characters, and numbers, then used the word segmentation tool to segment the food name into a collection of words. Since food name was a text field, we used word2vec, an approach that trains an $\mathrm{N}$-gram language model using a neural network and finds vectors corresponding to the words to learn high quality spatial representation of words from a large amount of unstructured text data [37], to embed food 
name information into vectors, using an open pretrained Chinese word embedding model [38] to represent the food name that trains text data from Baidu Encyclopedia. After mapping words into vectors, semantically similar words were relatively close in the vector space. To maintain the same dimension in each disease case, we calculated the average value of word vectors for each food name and obtained a 300-dimension vector for each food name field. Then, using variance for feature selection, we determined the final variance threshold and the dimension of the word vectors by comparing the model results under different thresholds to reduce the dimension of word vectors to control the feature dimension within a reasonable range and reduce the training time of model. In addition, we used $t$-distributed stochastic neighbor embedding to reduce the word vectors to 2 dimensions and used a scatter plot to represent word vectors of the top 5 foods (we removed unknown foods, mixed foods, multiple foods and other foods) with the highest frequency among the 19 types [39], shown in Figure 1. Finally, all features were combined into 349-dimension vectors.

Figure 1. Representation of word vectors of food names in 2 dimensions.

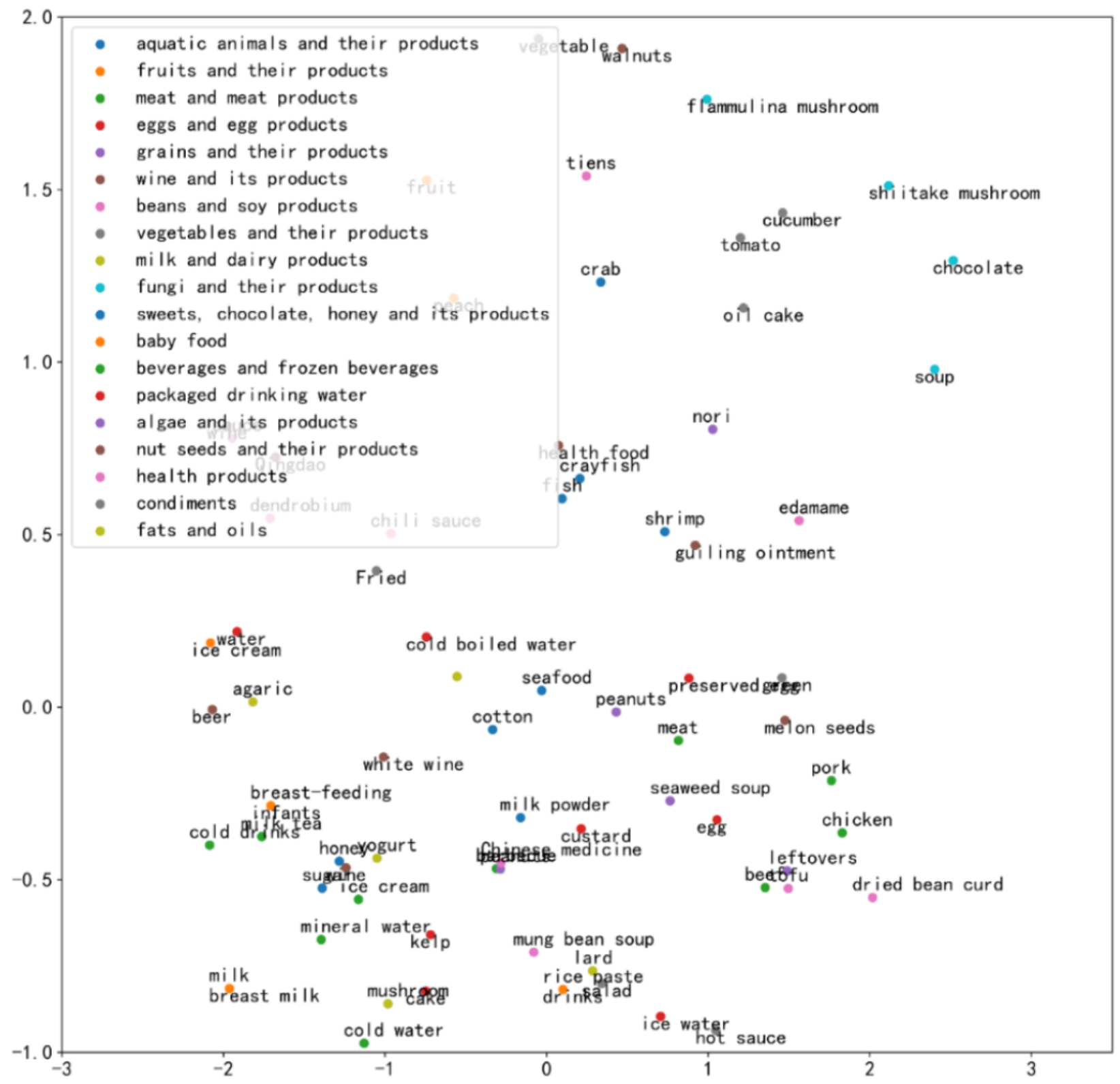

\section{Classification Methods}

Statistical analysis revealed the distribution of the 4 pathogens was relatively balanced; therefore, no extra sampling was required. We trained decision tree, random forest, gradient boost decision tree (GBDT), and adaptive boosting models with the processed data in Python (version 3.7; Scikit-learn package
[40]) and compared the results to obtain the best classification model.

Decision tree [41] is a nonparametric supervised learning method widely used in classification and regression. It differs from other classifiers that put all the features into the classifier at once. It decomposes the complex decision-making process into recursive steps, dividing the features. It does not require data normalization and has good interpretability [41]. 
Random forest is an ensemble model based on decision trees that can solve the problem of weak generalizability of decision trees [42]. It builds multiple decision trees and uses voting methods to obtain the final result. Each tree uses a replacement sampling method to obtain the training data and samples the features in a certain proportion. It can process high-dimensional data without feature selection. For unbalanced data sets, errors can be balanced; however, random forests may overfit on noisy data sets [42].

GBDT is also an integrated model based on decision trees [43]. Unlike random forest, which uses bagging to randomly select samples, GBDT uses the boosting method; it uses a serial training method to add the results of weak classifiers to obtain the prediction value. When training the next weak classifier, it fits the residual between the predicted value of the previous round of classifiers and the true value to improve the classification result.

Adaptive boosting is an integrated learning model that combines multiple weak classifiers into a strong classifier [44]. It can increase the weight of a sample that was misclassified by the previous weak classifier adaptively and train the next weak classifier. It has a better classification effect than a single decision tree [44].

\section{Training and Evaluation}

We divided 50,216 samples into training and test sets at a ratio of 7:3. The size of the training set was 35,151 samples, and the size of the test set was 15,065 samples. To tune the parameters, we used the grid search method. Specifically, we estimated the range of several important parameters in the model (such as the threshold of variance in feature selection, the number of weak classifiers, the depth of the tree, the minimal number of sample partitions, and the learning rate), and set a step size to obtain all the possible values of these parameters. The parameter combination that obtained the best model result was selected. In addition, we also used 10-fold cross-validation to improve the robustness of the model. Normalized confusion matrix, accuracy, macro-averaged precision (macro-P), macro-averaged recall (macro-R), and macro-averaged F1 score (macro-F1) were used to evaluate models. Multimedia Appendix 1 lists the evaluation criteria formulas.

\section{Feature Importance Evaluation}

In order to understand which features have a more important impact in the classification process, we calculated the importance value of each feature. The classification models we used were all based on tree structures, and the model of tree structures has natural advantages over other classification models in terms of interpretability. There are 2 ways to calculate the importance of features: Variable importance and Gini importance. Here, we used Gini importance to calculate the importance of features.

Gini importance is the degree to which the Gini index of a branch node formed by $M$ is calculated for a feature $M$ [45]. For the entire model, the average value of the Gini index of the feature on all trees is calculated. In the classification process based on tree structures, the faster the Gini index declines after a node splits, the greater the influence of the feature value represented by the split node on the classification result. The formula for Gini importance is shown as below.

$$
\begin{aligned}
& \operatorname{Gini}(\mathrm{D})=1-\sum_{i=1}^{n} p_{i}^{2} \\
& \Delta \operatorname{Gini}(\mathrm{M})=\operatorname{Gini}(D)-\operatorname{Gini}_{M}(D) \\
& \operatorname{Gini}_{M}(D)=\frac{\left|D_{1}\right|}{|D|} \operatorname{Gini}\left(D_{1}\right)+\frac{\left|D_{2}\right|}{|D|} \operatorname{Gini}\left(D_{2}\right)
\end{aligned}
$$

where $D$ represents the entire data set, and $p_{i}$ represents the probability of occurrence of each class. $\triangle \operatorname{Gini}(M)$ represents the decrease of impurity when adding the feature $M . D_{1}$ and $D_{2}$ represent the data set divided by feature $M$. The greater the value of $\triangle \operatorname{Gini}(M)$, the higher the feature importance.

\section{Results}

\section{Data Analysis}

Through the geographical distribution of the detection rate of pathogens (Figure 2), it can be seen that the geographical distribution of the detection rate of different pathogens is somewhat distinguishable. According to the detection rate of 4 pathogens in different months as shown in the upper left of Figure 3, it can be seen that there are some differences among the 4 pathogens in seasons or months. For example, $V$ parahaemolyticus occurs more frequently in summer, while Norovirus occurs more frequently in autumn and winter. Therefore, we can consider month as the time feature in data preprocessing. Through the distribution of age of patients of 4 pathogens (the upper right of Figure 3), the distribution trends of E coli, Salmonella, and Norovirus in different age groups are similar, and they were concentrated between 0 and 10 years old. Patients with Vparahaemolyticus were between 20 and 40 years old, which was different from the other 3 pathogens. The bottom left of Figure 3 shows the gender distribution and the bottom right of Figure 3 shows the distribution of 4 pathogens in 23 food categories. These analysis results show the difference among 4 pathogens. 
Figure 2. The geographic distribution of the detection rates of pathogens.
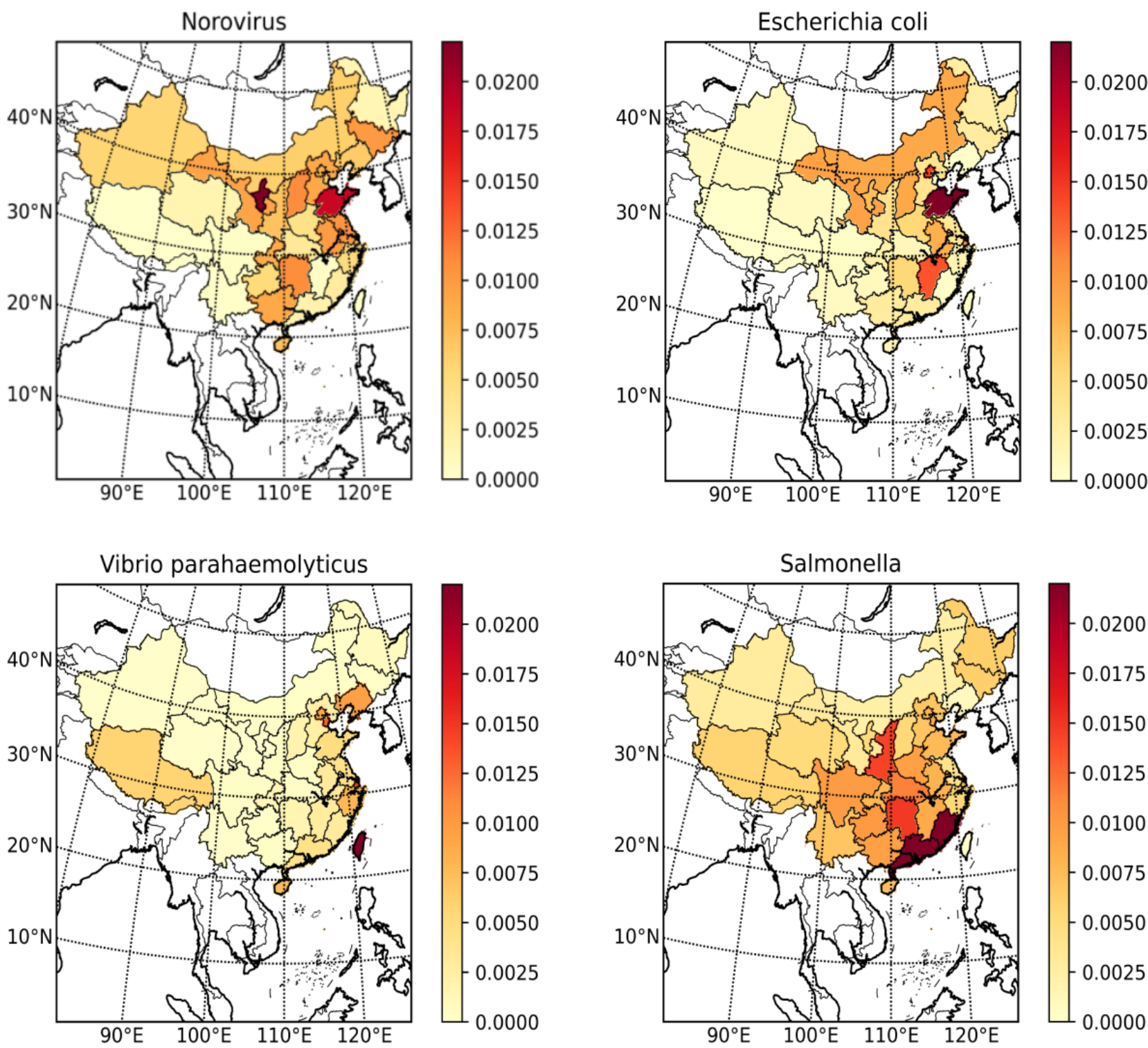
Figure 3. The distribution chart of the features of foodborne diseases. The upper left is the distribution of the detection rate of the pathogens by time; the upper right is the distribution of the pathogens by patient age; the bottom left is the distribution of the pathogens by patient gender; and the bottom right is the distribution of the pathogens by food type.
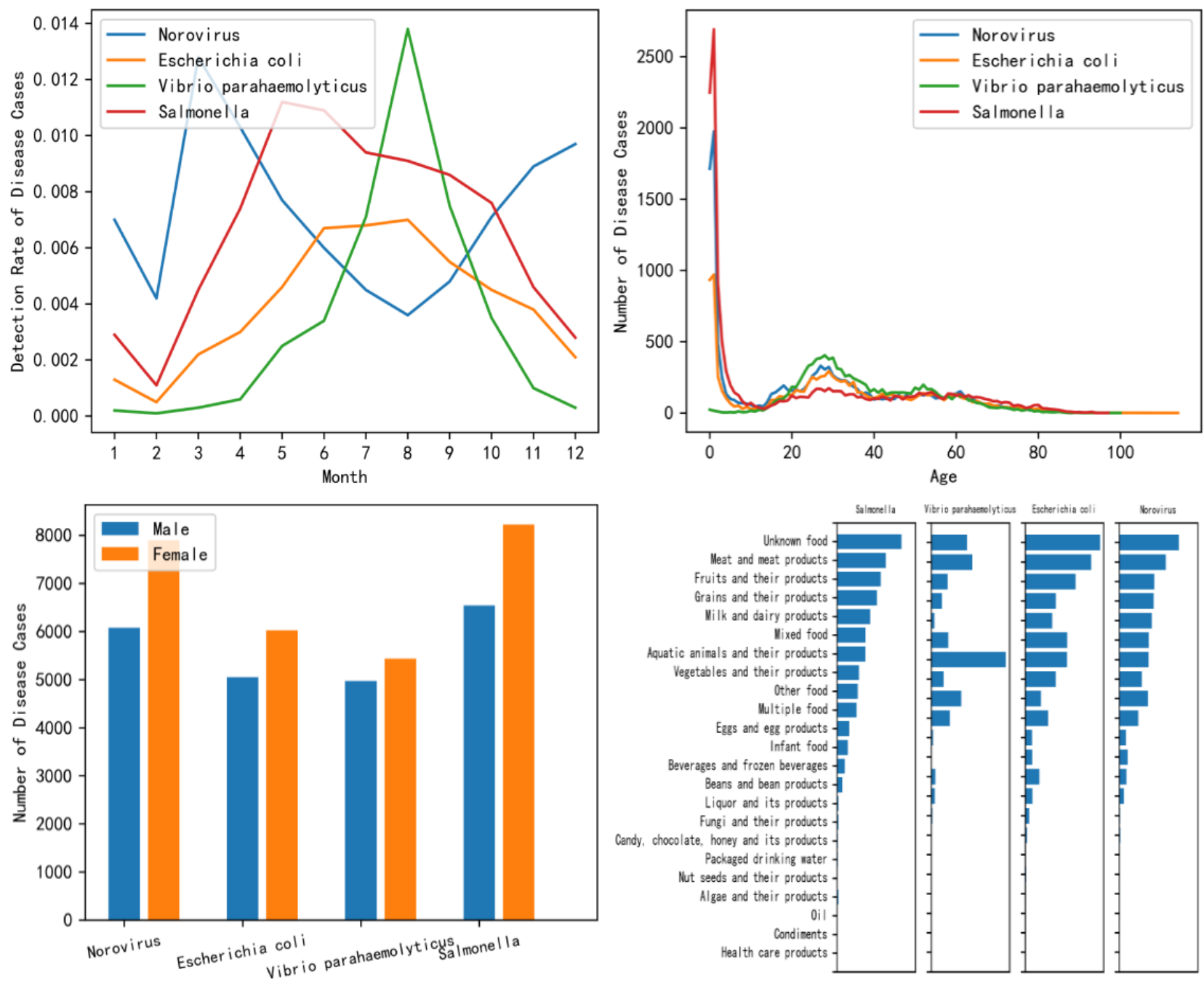

\section{Classification Results}

The decision tree model's performance was worse than the those of the other 3 integrated models; its accuracy, macro-P, macro-R, and macro-F1 rate were approximately 63\% (Table 4). Because the decision tree requires adjustment of fewer parameters and the model is relatively simple, we chose to use the decision tree model to perform feature selection and applied the results to the other models to reduce the number of parameters in those models that need to be adjusted. By comparing the model results under different variance thresholds, we found that increases in the word vector dimension did not greatly improve the effect of the model but increased the training time. Therefore, to balance the model effect and time cost, we finally retained a 30-dimensional word vector feature.

Each tree in the random forest model used replaceable data and feature sampling, and decision trees were parallel. The classification results were better than those for a single decision tree. After adjusting the number of decision trees, the depth of the tree, and the minimum number of split samples, the average

accuracy of the random forest model was $1 \%$ higher than that of the decision tree model.

The classification results of the GBDT model were better than those of the other models. When training the GBDT model, we set the size of feature set to 0.8 , which means that each single decision tree in GBDT only selects $80 \%$ of the features for training, to ensure that each training process focused on different combinations of features. After parameter tuning (weak classifier: 171 ; depth of the tree: 20 ; minimum number of sample partitions: 50), an accuracy of $69 \%$ was achieved.

Adaptive boosting reach an accuracy of approximately 67\%, only lower than that of the GBDT model.

The classification recalls of the 4 pathogens (Norovirus, E coli, $V$ parahaemolyticus, Salmonella) were $69 \%, 60 \%, 73 \%$, and $69 \%$, respectively (Table 5). Among misclassified E coli samples, approximately $17 \%$ of the samples were misclassified as Norovirus, $10 \%$ of the samples were misclassified as $V$ parahaemolyticus, and $13 \%$ of the samples were misclassified as Salmonella. 
Table 4. The classification results of 4 classification models.

\begin{tabular}{lllll}
\hline & Macro- $\mathrm{P}^{\mathrm{a}}$ & Macro- $\mathrm{R}^{\mathrm{b}}$ & Macro-F1 $^{\mathrm{c}}$ & Accuracy \\
\hline Decision tree & 0.62 & 0.63 & 0.63 & 0.63 \\
Random forest & 0.63 & 0.64 & 0.64 & 0.64 \\
GBDT $^{\mathrm{d}}$ & 0.68 & 0.69 & 0.69 & 0.69 \\
AdaBoost $^{\mathrm{e}}$ & 0.67 & 0.66 & 0.67 & 0.67 \\
\hline
\end{tabular}

${ }^{\mathrm{a}}$ Macro-P: macro-averaged precision.

${ }^{\mathrm{b}}$ Macro-R: macro-averaged recall.

${ }^{\mathrm{c}}$ Macro-F1: macro-averaged F1 score.

${ }^{\mathrm{d}}$ GBDT: gradient boost decision tree.

${ }^{\mathrm{e}}$ AdaBoost: adaptive boosting.

Table 5. Normalized confusion matrix of classification result in the GBDT model.

\begin{tabular}{lllll}
\hline Actual & $\begin{array}{l}\text { Predicted } \\
\text { Norovirus }\end{array}$ & E coli & V parahaemolyticus & Salmonella \\
\hline Norovirus & 0.69 & 0.13 & 0.06 & 0.13 \\
E coli & 0.17 & 0.60 & 0.10 & 0.13 \\
V parahaemolyticus & 0.05 & 0.12 & 0.73 & 0.10 \\
Salmonella & 0.12 & 0.10 & 0.09 & 0.69 \\
\hline
\end{tabular}

\section{Feature Importance Evaluation}

For the 4 classifiers, the top 10 important features of each classifier are shown in Table 6.

According to Table 6, we can see that the 4 classifiers have higher feature importance values in the longitude and latitude of the geographical location, the time of illness, the age of patient, the name of food, and certain symptoms (such as fever, frequency of diarrhea, frequency of vomiting). This means that these attributes have a great influence on the discrimination of pathogens. In addition, GBDT, decision tree, and AdaBoost also have relatively high importance value on diarrhea traits, and the stomachache symptom has a high impact on the classification process of the AdaBoost model and the random forest model. In the food types, aquatic animals and their products had a high impact on the classification process using decision tree or random forest. Combined with the previous exploratory analysis of data distribution, we can find that the attributes with large differences in data distribution have larger attribute importance values too.

Table 6. The top 10 important features in the 4 classifiers.

\begin{tabular}{lllll}
\hline Importance rank & Decision tree & Random forest & GBDT $^{\mathrm{a}}$ & AdaBoost $^{\mathrm{b}}$ \\
\hline 1 & Latitude & Sick time & Latitude & Latitude \\
2 & Sick time & Latitude & Longitude & Longitude \\
3 & Longitude & Longitude & Sick time & Sick time \\
4 & Age of patients & Age of patients & Diarrhea frequency & Age of patients \\
5 & Fever & Fever & Age of Patients & Diarrhea Frequency \\
6 & Vomiting frequency & Aquatic animals and their & Diarrhea traits & Food name \\
7 & products & Food name & Diarrhea frequency \\
8 & Diarrhea frequency & Vomiting frequency & Vomiting frequency & Diarrhea traits \\
9 & Food name & Diarrhea frequency & Fever & Fever \\
\end{tabular}

${ }^{\mathrm{a}}$ GBDT: gradient boost decision tree.

${ }^{\mathrm{b}}$ AdaBoost: adaptive boosting. 


\section{Discussion}

\section{Principal Results}

We used foodborne disease case data to visually analyze several features of foodborne diseases, and we found that the analysis results were consistent with those of previous studies in some aspects. For example, Norovirus occurs more frequently in autumn and winter [46], and distribution trends of patients' age of E coli, Salmonella, and Norovirus are concentrated between 0 and 10 years old, which is consistent with a study result that young children are more susceptible to foodborne diseases [5]. Besides, for the 4 foodborne pathogens, there were differences in geographical, time of illness, patients' age, patients' gender, and exposed food categories distribution.

Of the 4 machine learning methods that we used, the best-performing classification model was the GBDT model with a classification accuracy up to $69 \%$ with the optimal parameters being 171 weak classifiers, depth of the tree-20, and minimum number of sample partitions-50, the dimension of word vector of food name-30. The classification recall of $V$ parahaemolyticus was the highest, reaching almost $73 \%$, while for $E$ coli, it was only $60 \%$. The model was most likely to mistake Norovirus for $E$ coli. Based on this result, it can be reasonably inferred that the $V$ parahaemolyticus is different from the other 3 pathogens (with respect to disease case information), and E coli and Norovirus may have similarities in distribution areas, time of illness, disease symptoms, and patient information.

We found that the 4 classifiers have higher feature importance values for time of illness, geographical longitude and latitude, and patient age. The optimal GBDT model had higher feature importance values in terms of diarrhea frequency, food name, and diarrhea traits. This result is consistent with the previous data analysis to a certain extent, such as the distribution of the 4 pathogens in geographical space, time, and patient age is quite different, so it further proves that our method is reasonable.

\section{Primary Contribution}

Supervised learning was conducted to extract distinguishable features of different pathogens, then we compared the results of multiple experiments to obtain the optimal classification model for predicting possible pathogens for cases with unknown pathogens. The classification accuracy of the optimal model for Salmonella, Norovirus, E coli, and V parahaemolyticus can reach $69 \%$. The model also has good scores on other evaluation indicators. Our contributions can be summarized as below:

1. We proposed a machine learning model that can automatically predict pathogens without laboratory testing. This model can potentially reduce the burden of demand for domain knowledge and technical equipment.

2. We conducted a formal analysis of the relationship between pathogens and several features of disease cases. This approach help find some distinguishable features of different pathogens.

3. Our approach can assist doctors to quickly identify the pathogens of foodborne diseases, especially if there is no sufficient test equipment and budget. This can help doctors give specific medical treatment for foodborne diseases caused by different pathogens and provide support for more accurate diseases burden estimation. It may also lead to a more accurate foodborne disease outbreak prediction.

\section{Limitations}

This study had certain limitations. First, it should be noted that the disease case data come from a surveillance platform, and results are, therefore, influenced by the quality of the surveillance platform data-though the data were confirmed cases from hospitals or the $\mathrm{CDC}$, and thus very reliable, the scope was limited. Many people may choose to buy nonprescription drugs rather than go to the hospital for treatment when their illness is not as severe; therefore, the number of disease cases collected in the surveillance platform may be lower than the actual value [14]. To solve this problem, aggregating other data sources, such as social network data or search engine data, is a useful solution. Second, a large number of patients were between 0 and 10 years old. Although some studies have shown that the burden of disease caused by foodborne disease is higher in young children [46], it has not excluded that children have a higher probability of visiting a doctor after illness than adults. Third, in the geographical distribution of pathogens, there were some differences for the 4 pathogens, but distribution may be affected by population size and economic status. For example, the population and economic conditions in the eastern part of China are better than those in western part, thus the incidence rate in the east may be higher than that in the west.

\section{Conclusions}

We presented a machine learning-based classification method for pathogens of foodborne diseases using the case data of foodborne diseases in the National Foodborne Disease Surveillance Reporting System. Our optimal model achieved a $69 \%$ classification accuracy rate on Salmonella, Norovirus, E coli, and V parahaemolyticus. Pathogens are the main cause of foodborne diseases, research on pathogens is essential for foodborne diseases; however, due to the time and technical limitations, pathogen detection is generally performed in only a few cases, causing difficulty for identification and diagnosis of diseases. We proposed a classification method that can predict pathogens of diseases without laboratory testing. Although this method cannot replace traditional laboratory testing, it can be used to assist traditional identification with little time cost and equipment requirements. This method can help to quickly identify and diagnose foodborne disease and offer some guidance for specific medical treatments for foodborne diseases caused by different pathogens. In addition, it can also provide some support for improving accuracy rate in further foodborne diseases burden estimation and outbreak prediction.

In the future, we plan to compare our results with data from the foodborne disease outbreak surveillance system for optimization guidance, and we will try to add other domain knowledge or refer to other data sources to get more reliable results. In addition, we will carry out disease outbreak prediction. 


\section{Acknowledgments}

This research is supported by the National Key Research and Development Plan (grant number 2017YFC1601504) and the Natural Science Foundation of China (grant number 61836013).

\section{Conflicts of Interest}

The authors declare that they have no conflict of interest.

\section{Multimedia Appendix 1}

Evaluation criteria.

[DOCX File, 13 KB-Multimedia Appendix 1]

\section{References}

1. Dodd C, Aldsworth T, Stein R, Cliver D, Riemann H. In: Jones JL, editor. Foodborne Diseases. Netherlands: Academic Press; 2017.

2. Bean H, Griffin P, Goulding JS. Foodborne disease outbreaks, 5-year summary, 1983-1987. Journal of Food Protection 1990;53(8):711-728. [doi: 10.4315/0362-028x-53.8.711]

3. Oliver SP. Foodborne pathogens and disease special issue on the national and international PulseNet network. Foodborne Pathog Dis 2019 Jul;16(7):439-440. [doi: 10.1089/fpd.2019.29012.int] [Medline: 31259613]

4. Liu J, Bai L, Li W, Han H, Fu P, Ma X, et al. Trends of foodborne diseases in China: lessons from laboratory-based surveillance since 2011. Front Med 2018 Feb 27;12(1):48-57. [doi: 10.1007/s11684-017-0608-6] [Medline: 29282610]

5. Kirk MD, Pires SM, Black RE, Caipo M, Crump JA, Devleesschauwer B, et al. World Health Organization estimates of the global and regional disease burden of 22 foodborne bacterial, protozoal, and viral diseases, 2010: a data synthesis. PLoS Med 2015 Dec 3;12(12):e1001921 [FREE Full text] [doi: 10.1371/journal.pmed.1001921] [Medline: 26633831]

6. Centers for Disease Control and Prevention. Foodborne Diseases Active Surveillance Network, 1996. MMWR Morb Mortal Wkly Rep 1997 Mar 28;46(12):258-261 [FREE Full text] [Medline: 9087688]

7. Foodborne Disease Monitoring and Reporting System. National Center for Food Safety Risk Assessment. URL: https:/ /foodnet.cfsa.net.cn/ [accessed 2021-01-16]

8. Oldroyd RA, Morris MA, Birkin M. Identifying methods for monitoring foodborne illness: review of existing public health surveillance techniques. JMIR Public Health Surveill 2018 Jun 06;4(2):e57 [FREE Full text] [doi: 10.2196/publichealth.8218] [Medline: 29875090]

9. Scallan E, Hoekstra RM, Angulo FJ, Tauxe RV, Widdowson M, Roy SL, et al. Foodborne illness acquired in the United States-major pathogens. Emerg Infect Dis 2011 Jan;17(1):7-15. [doi: 10.3201/eid1701.p11101]

10. Mandal P, Biswas A, Choi K, Pal U. Methods for rapid detection of foodborne pathogens: an overview. American Journal of Food Technology 2011 Jan 15;6(2):87-102. [doi: 10.3923/ajft.2011.87.102]

11. Law JW, Ab Mutalib N, Chan K, Lee L. Rapid methods for the detection of foodborne bacterial pathogens: principles, applications, advantages and limitations. Front Microbiol 2014 Jan 12;5:770 [FREE Full text] [doi:

10.3389/fmicb.2014.00770] [Medline: 25628612]

12. Naravaneni R, Jamil K. Rapid detection of food-borne pathogens by using molecular techniques. J Med Microbiol 2005 Jan;54(Pt 1):51-54. [doi: 10.1099/jmm.0.45687-0] [Medline: 15591255]

13. Pan W, Zhao J, Chen Q. Classification of foodborne pathogens using near infrared (NIR) laser scatter imaging system with multivariate calibration. Sci Rep 2015 Apr 10;5:9524 [FREE Full text] [doi: 10.1038/srep09524] [Medline: 25860918]

14. Flint JA, Van Duynhoven YT, Angulo FJ, DeLong SM, Braun P, Kirk M, et al. Estimating the burden of acute gastroenteritis, foodborne disease, and pathogens commonly transmitted by food: an international review. Clin Infect Dis 2005 Sep 01;41(5):698-704. [doi: 10.1086/432064] [Medline: 16080093]

15. Kuehn BM. Agencies use social media to track foodborne illness. JAMA 2014 Jul 09;312(2):117-118. [doi: 10.1001/jama.2014.7731] [Medline: 24963655]

16. Sadilek A, Kautz H, DiPrete L, Labus B, Portman E, Teitel J, et al. Deploying Nemesis: preventing foodborne illness by data mining social media. AIMag 2017 Mar 31;38(1):37-48. [doi: 10.1609/aimag.v38i1.2711]

17. Effland T, Lawson A, Balter S, Devinney K, Reddy V, Waechter H, et al. Discovering foodborne illness in online restaurant reviews. J Am Med Inform Assoc 2018 Dec 01;25(12):1586-1592 [FREE Full text] [doi: 10.1093/jamia/ocx093] [Medline: 29329402]

18. Nogueira M, Greis N. Rule-based complex event processing for food safety and public health. Berlin, Heidelberg: Springer; 2011 Presented at: International Workshop on Rules and Rule Markup Languages for the Semantic Web; November 3-5; Fort Lauderdale, FL, USA. [doi: 10.1007/978-3-642-22546-8 31]

19. Bean N, Goulding J, Lao C. Surveillance for foodborne disease outbreaks-United States, 1988-1992. J Food Prot 1997;60(10):1265-1286. [doi: $10.4315 / 0362-028 x-60.10 .1265]$ 
20. Lynch M, Painter J, Woodruff R, Braden C, Centers for Disease Control and Prevention. Surveillance for foodborne-disease outbreaks--United States, 1998-2002. MMWR Surveill Summ 2006 Nov 10;55(10):1-42 [FREE Full text] [Medline: $\underline{17093388]}$

21. Swaminathan B, Barrett TJ, Hunter SB, Tauxe RV. PulseNet: the molecular subtyping network for foodborne bacterial disease surveillance, United States. Emerg Infect Dis 2001 Jun;7(3):382-389. [doi: 10.3201/eid0703.017303]

22. Allard MW, Strain E, Melka D, Bunning K, Musser SM, Brown EW, et al. Practical value of food pathogen traceability through building a whole-genome sequencing network and database. J Clin Microbiol 2016 Mar 23;54(8):1975-1983. [doi: 10.1128/jcm.00081-16]

23. Hendriksen RS, Vieira AR, Karlsmose S, Lo Fo Wong DM, Jensen AB, Wegener HC, et al. Global monitoring of Salmonella serovar distribution from the World Health Organization Global Foodborne Infections Network Country Data Bank: results of quality assured laboratories from 2001 to 2007. Foodborne Pathog Dis 2011 Aug;8(8):887-900. [doi:

10.1089/fpd.2010.0787] [Medline: 21492021]

24. Liu X, Chen Y, Wang X, Ji R. [Foodborne disease outbreaks in China from 1992 to 2001 national foodborne disease surveillance system]. Wei Sheng Yan Jiu 2004 Nov;33(6):725-727. [Medline: 15727189]

25. Liu X, Chen Y, Fan Y, Wang M. [Foodborne diseases occurred in 2003--report of the National Foodborne Diseases Surveillance System, China]. Wei Sheng Yan Jiu 2006 Mar;35(2):201-204. [Medline: 16758972]

26. Chen Y, Guo Y, Wang Z, Liu X, Liu H, Dai Y, et al. [Foodborne disease outbreaks in 2006 report of the National Foodborne Disease Surveillance Network, China]. Wei Sheng Yan Jiu 2010 May;39(3):331-334. [Medline: 20568464]

27. Wallace D, Van Gilder T, Shallow S, Fiorentino T, Segler SD, Smith KE, et al. Incidence of foodborne illnesses reported by the foodborne diseases active surveillance network (FoodNet)-1997. FoodNet Working Group. J Food Prot 2000 Jun;63(6):807-809. [doi: 10.4315/0362-028x-63.6.807] [Medline: 10852576]

28. Dewey-Mattia D, Manikonda K, Hall AJ, Wise ME, Crowe SJ. Surveillance for foodborne disease outbreaks - United States, 2009-2015. MMWR Surveill Summ 2018 Jul 27;67(10):1-11 [FREE Full text] [doi: 10.15585/mmwr.ss6710a1] [Medline: $\underline{\text { 30048426] }}$

29. Xiao X, Ge Y, Guo Y. Automated detection for probable homologous foodborne disease outbreaks. 2015 Presented at: Pacific-Asia Conference on Knowledge Discovery and Data Mining; May 19-22; Ho Chi Minh, Vietnam. [doi: 10.1007/978-3-319-18038-0 44]

30. Jordan MI, Mitchell TM. Machine learning: Trends, perspectives, and prospects. Science 2015 Jul 17;349(6245):255-260. [doi: 10.1126/science.aaa8415] [Medline: 26185243]

31. Aramaki E, Maskawa S, Morita M. Twitter Catches the Flu: Detecting Influenza Epidemics Using Twitter. In: Proceedings of the Conference on Empirical Methods in Natural Language Processing.: Association for Computational Linguistics; 2011 Presented at: Conference on Empirical Methods in Natural Language Processing; July 27-31; Edinburgh, Scotland.

32. Friedman JH, Meulman JJ. Multiple additive regression trees with application in epidemiology. Stat Med 2003 May 15;22(9):1365-1381. [doi: 10.1002/sim.1501] [Medline: 12704603]

33. Teyhouee A, McPhee-Knowles S, Waldner C. Prospective detection of foodborne illness outbreaks using machine learning approaches. 2017 Presented at: International Conference on Social Computing, Behavioral-Cultural Modeling and Prediction and Behavior Representation in Modeling and Simulation; July 5-8; Washington DC, USA. [doi: 10.1007/978-3-319-60240-0 36]

34. Vilne B, Meistere I, Grantina-Ievina L, Kibilds J. Machine learning approaches for epidemiological investigations of food-borne disease outbreaks. Front Microbiol 2019 Aug 6;10:1722 [FREE Full text] [doi: 10.3389/fmicb.2019.01722] [Medline: $\underline{31447800]}$

35. Thakur M, Olafsson S, Lee J, Hurburgh CR. Data mining for recognizing patterns in foodborne disease outbreaks. Journal of Food Engineering 2010 Mar;97(2):213-227. [doi: 10.1016/j.jfoodeng.2009.10.012]

36. D'Souza RM, Becker NG, Hall G, Moodie KBA. Does ambient temperature affect foodborne disease? Epidemiology 2004 Jan;15(1):86-92. [doi: 10.1097/01.ede.0000101021.03453.3e] [Medline: 14712151]

37. Mikolov T, Sutskever I, Chen K, Corrado GS, Dean J. Distributed representations of words and phrases and their compositionality. Advances in Neural Information Processing Systems 2013;26 [FREE Full text] [doi: $10.5555 / 2999792.2999959]$

38. Li S, Zhao Z, Hu R. Analogical reasoning on Chinese morphological and semantic relations. In: Proceedings of the 56th Annual Meeting of the Association for Computational Linguistics. 2018 Presented at: 56th Annual Meeting of the Association for Computational Linguistics; July 15-20; Melbourne, Australia. [doi: 10.18653/v1/p18-2023]

39. Maaten L, Hinton G. Visualizing Data using t-SNE. J Mach Learn Res 2008;9(11):2579-2605.

40. Varoquaux G, Buitinck L, Louppe G, Grisel O, Pedregosa F, Mueller A. Scikit-learn. GetMobile: Mobile Comp and Comm 2015 Jun;19(1):29-33. [doi: 10.1145/2786984.2786995]

41. Safavian S, Landgrebe D. A survey of decision tree classifier methodology. IEEE Trans Syst Man Cybern 1991;21(3):660-674. [doi: $10.1109 / 21.97458$ ]

42. Ho T. Random decision forests. In: Proceedings of 3rd International Conference on Document Analysis and Recognition. 1995 Presented at: 3rd International Conference on Document Analysis and Recognition; Aug 14; Montreal, Quebec, Canada. [doi: 10.1109/icdar.1995.598994] 
43. Friedman JH. Greedy function approximation: a gradient boosting machine. Ann. Statist 2001 Oct;29(5):1189-1232. [doi: 10.1214/aos/1013203451]

44. Freund Y, Schapire RE. A decision-theoretic generalization of on-line learning and an application to boosting. Journal of Computer and System Sciences 1997 Aug;55(1):119-139. [doi: 10.1006/jcss.1997.1504]

45. Gordon A, Breiman L, Friedman J, Olshen R, Stone C. Classification and regression trees. Biometrics 1984 Sep;40(3):874. [doi: 10.2307/2530946]

46. Ahmed SM, Lopman BA, Levy K. A systematic review and meta-analysis of the global seasonality of norovirus. PLoS One 2013 Oct 2;8(10):e75922 [FREE Full text] [doi: 10.1371/journal.pone.0075922] [Medline: 24098406]

\section{Abbreviations \\ CDC: Centers for Disease Control \\ GBDT: gradient boost decision tree \\ Macro-F1: macro-averaged F1 score \\ Macro-P: macro-averaged precision \\ Macro-R: macro-averaged recall \\ WHO: World Health Organization}

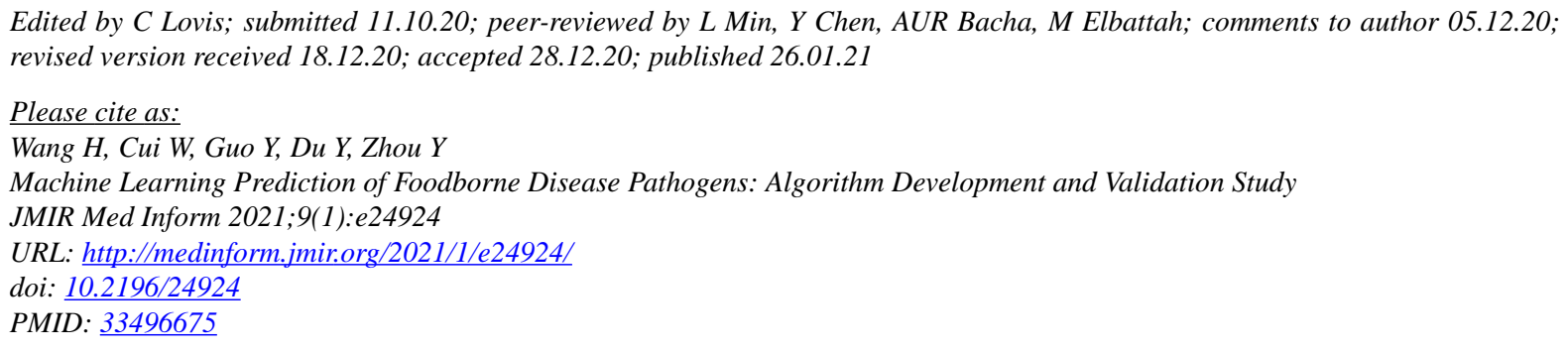

CHanxue Wang, Wenjuan Cui, Yunchang Guo, Yi Du, Yuanchun Zhou. Originally published in JMIR Medical Informatics (http://medinform.jmir.org), 26.01.2021. This is an open-access article distributed under the terms of the Creative Commons Attribution License (https://creativecommons.org/licenses/by/4.0/), which permits unrestricted use, distribution, and reproduction in any medium, provided the original work, first published in JMIR Medical Informatics, is properly cited. The complete bibliographic information, a link to the original publication on http://medinform.jmir.org/, as well as this copyright and license information must be included. 